\title{
Safety of Parenteral Third-Generation Cephalosporins
}

F. Robert FekeTY, M.D. Ann Arbor, Michigan

Knowledge of side effects associated with different cephalosporins may be of help to prescribers. There are several side effects that are common to all cephalosporins, but overall, cefotaxime and ceftizoxime cause the fewest adverse reactions. Bleeding is probably the most common serious side effect of cephalosporins. Moxalactam causes coagulopathy and bleeding more often than do other cephalosporins, probably because it is carboxylated and has a methylthiotetrazole side chain. Cefoperazone also has a methylthiotetrazole side chain and may cause bleeding, particularly when used in doses greater than $4 \mathrm{~g}$ per day. Ceftriaxone has a similar side chain and there is some evidence that it can induce a coagulopathy. Coagulation tests should be monitored when any of the third-generation cephalosporins are given to patients with a high risk of bleeding. Disulfiram-like reactions are also related to the side chains associated with coagulation defects and have been reported when patients receiving cefoperazone, moxalactam, or ceftriaxone have ingested alcohol. Seizures have been reported with ceftazidime, but are uncommon. Hematologic reactions are rare with all third-generation cephalosporins. Benign diarrhea and Clostridium difficile colitis probably occur most often with moxalactam, cefoperazone, ceftazidime, and ceftriaxone, but there are few good data on this issue. Ceftriaxone has the unique ability to cause sludge (also referred to as pseudolithiasis) to form in the gallbladder, particularly in children. This may be associated with nausea, anorexia, epigastric distress, and colic, and is usually detected using ultrasonography. The sludge dissolves and symptoms subside after therapy is discontinued. None of the third-generation cephalosporins is clearly significantly nephrotoxic, even when combined with aminoglycosides. Most of the third-generation cephalosporins have surprisingly few serious side effects, which make them attractive for use in the treatment of a wide variety of serious infections.

From the Division of Infectious Diseases, Department of Internal Medicine, University of Michigan Hospitals, Ann Arbor, Michigan. Requests for reprints should be addressed to Dr. F Robert Fokety. Division of Infectious Diseases. Department of Inter nal Medicine, University of Michigan Hospitals, 3116 Taubman Center, Ann Arbor, Michigan 48109-0378.
$\mathbf{T}$ hird-generation cephalosporins have a remarkably broad spectrum of antibacterial activity, and all of those currently available are administered via the parenteral route. They are, therefore, attractive for empiric therapy of serious or polymicrobial infections. The six third-generation cephalosporins that will be discussed in this review are cefotaxime (Claforan), ceftizoxime (Cefizox), ceftriaxone (Rocephin), moxalactam (Moxam, Latamoxef), cefoperazone (Cefobid), and ceftazidime (Fortaz, Ceftaz, Tazicef). Extensive experience has shown that these agents are relatively free of serious side effects, which is remarkable in view of the confounding influence of the disease-related adverse effects similar to those commonly attributed to the use of these antimicrobials [1] The overall rate of adverse effects is low and similar for all third-generation cephalosporins $[1,2]$, particularly when compared with the aminoglycosides. The cephalosporins show slightly different side effect profiles, some of which are related to differences in molecular structure (e.g., coagulopathies resulting from the presence of the N-methylthiotetrazole [MTT] side chain). Other side effects are related to the broadspectrum antibacterial activity of the cephalosporins or to their excretion in large amounts into the gastrointestinal tract, with resultant marked changes in the microbial ecology of the bowel flora. Not surprisingly, there are some important differences between these antimicrobials in the frequency and nature of these adverse effects, and knowledge of these can be helpful to clinicians wishing to prevent or treat these complications.

All of the third-generation cephalosporins can cause local pain on administration, phlebitis, fever, pruritus, rashes, anorexia, nausea, vomiting, nonspecific diarrhea, abnormal liver function tests, and positive direct Coomb's antiglobulin tests on serum. These side effects are usually mild and not serious, since they frequently subside promptly when the drug treatments are discontinued. All of these antimicrobials are capable of causing serious allergic reactions, and should, therefore, be used with caution in patients who have previously had reactions to beta-lactam antimicrobials.

\section{COAGULATION DEFECTS AND BLEEDING}

Coagulopathies and bleeding are probably the most serious side effects caused by the third-generation cephalosporins. The most common mechanisms behind cephalosporin-induced coagulation deficits or bleeding are shown in Table I $[3,4]$. Most cases of bleeding associated with these compounds are caused by either vitamin K-responsive hypoprothrombinemia, as manifested by prolongation of the prothrombin time, or an acquired platelet functional defect, as manifested by an increase in bleeding time. 


\begin{tabular}{|l|}
\hline TABLE I \\
Mechanisms of Cephalosporin-Induced Coagulation Deficits \\
or Bleeding \\
\hline \\
- Induction of vitamin K-responsive hypoprothrombinemia \\
- Thromboction of an acquired platelet defeect \\
Immune secondary to bone marrow suppression \\
- Inhibition of fibrin polymimerization \\
- Potentiation of the effects of coumadin
\end{tabular}

\section{TABLE II}

Number of Patients Reported to the United States Food and Drug Administration between 1981 and 1987 as Having Gastrointestinal Bleeding Resulting from Parenteral Treatment with Third-Generation Cephalosporins

\begin{tabular}{lr}
\hline Cefotaxime & 19 \\
Ceftriaxone & 6 \\
Cefoperazone & 21 \\
Ceftazidime & 26 \\
Moxalactam & 216 \\
Ceftizoxime & - \\
\hline
\end{tabular}

The relative frequency of coagulopathies and bleeding is not accurately documented for the different third-generation cephalosporins. An analysis of reports (as sent to the United States Food and Drug Administration between 1981 and 1987) is shown in Table II [4]. The numbers of patients treated with each agent were not reported. Approximately 25 percent of these patients who were reported to the Food and Drug Administration died, and though the cause of death was not necessarily bleeding, these data clearly indicate that coagulopathy and bleeding are serious complications of the use of cephalosporins.

The lack of good comparative data is in part because in addition to the use of antimicrobials, these patients may also have important risk factors for bleeding, including malnutrition, poor dietary intake of vitamin $\mathrm{K}$, use of nasogastric suction, gastrointestinal or hepatic disease, or renal disease, or they may undergo surgical procedures or prolonged intravenous therapy without vitamin $\mathrm{K}$ supplementation [5].

Bleeding can occur with all the cephalosporins, but it appears that coagulopathies and bleeding are most frequent when the cephalosporin molecule contains a MTT side chain [6]. For third-generation cephalosporins, this moiety is found in both moxalactam [7-9] and cefoperazone $[10,11]$. Ceftriaxone contains a related structure, N-methylthiotriazone (MTZ) (Figure 1), the significance of which, with respect to coagulation defects, remains controversial. Nonetheless, more cases of bleeding, particularly from the gastrointestinal tract, have been reported in association with moxalactam than with any other widely used cephalosporin for which good data are available [4]. This probably results from adverse effects on both prothrombin synthesis and platelet function, as discussed in the following sections.

\section{ANTIBIOTIC-INDUCED HYPOPROTHROMBINEMIA}

Vitamin $\mathrm{K}$ is a cofactor in the hepatic microsomal carboxylation of the precursors of prothrombin (clotting factor II) known as convertin (factor VII), antihe-

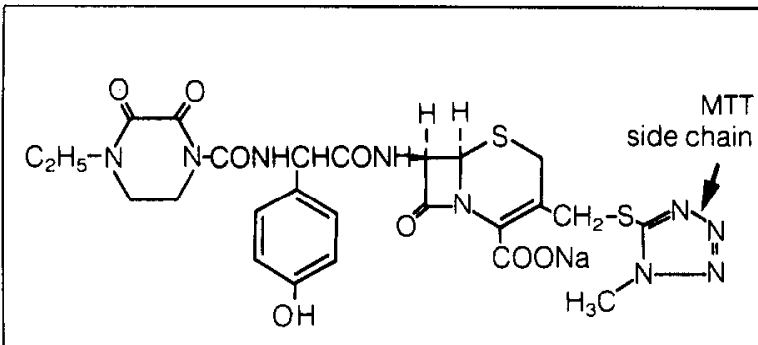

Cefoperazone<smiles>CC(=O)OCC1=C(C(=O)O[Na])N2C(=O)[C@H](NC(=O)c3csc(N)n3)[C@H]2SC1</smiles>

Cefotaxime

Ceftazidime<smiles>CC(=O)ON=C(C(=O)OC(C)=O)c1csc(N)n1</smiles><smiles>CNOC(=O)C(=NOC)c1csc(N)n1</smiles>

Ceftizoxime

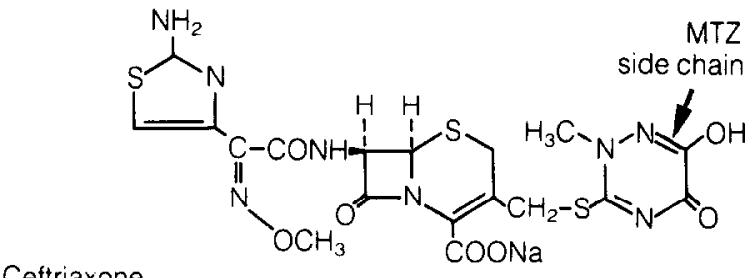

Ceftriaxone

MTT

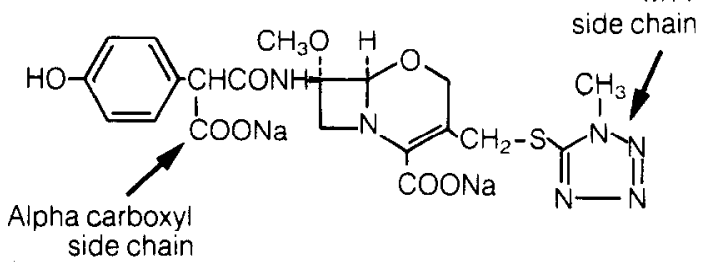

Moxalactam

Figure 1. Structures of various third-generation cephalosporins. Note the presence of the MTT side chain on both the cefoperazone and moxalactam molecules. These are associated with bleeding from the gastrointestinal tract. The role of the related MT group on the ceftriaxone molecule with respect to coagulation defects is unknown. The alpha-carboxyl group on moxalactam is associated with a functional defect in platelets; the oxygen in the secondary ring structure probably facilitates splitting of the MTT side chain, thus permitting its enterohepatic recirculation. 


\section{TABLE III}

\section{Characteristics of Immune Hematologic Adverse Reactions} following Administration of Third-Generation Cephalosporins

\section{- Relatively uncommon}

- Produces a positive Coomb's antiglobulin test (3 percent of patients?)

- Hemolytic anemia known to be caused by ceftriaxone

- Leukopenia is induced by high-dose therapy for 2-3 weeks with cephalosporins. This is rapidly reversible

- Thrombocytopenia is rare, but can occur with ceftriaxone and cefotaxime

mophilic factor B (factor IX), and Stuart-Prower factor (factor $\mathrm{X}$ ). These clotting factors must undergo carboxylation in order to bind to phospholipid surfaces and to contribute to the formation of a fibrin clot at a bleeding point [12]. During carboxylation, vitamin $K$ is inactivated and must be replaced or reactivated to allow continued conversion of precursors to the carboxylated form. Vitamin $K_{1}$ (phylloquinine) is the primary source of vitamin $\mathrm{K}$ for humans, and is derived from dietary sources, whereas vitamin $\mathrm{K}_{2}$ (menoquinone) is produced by micro-organisms within the lumen of the colon. Such microbial vitamin $K_{2}$ becomes a highly important source of vitamin $K$ when dietary sources are deficient. Malnourished, vitamin K-deficient patients receiving parenteral feeding without vitamin $\mathrm{K}$ supplementation are highly dependent on these endogenous sources, and are, therefore, susceptible to bleeding, owing to antibiotic-induced reductions in the numbers of coliforms and Bacteroides in the colon. Both of these groups of organisms are of major importance in the synthesis of vitamin $\mathrm{K}_{2}$ [13]. It is possible, therefore, for cephalosporin antibiotics to interfere with the generation of clotting factors in two ways: (1) by affecting intestinal synthesis of vitamin $\mathrm{K}_{2}$; and (2) by impairing the carboxylation of vitamin $\mathrm{K}$ in the liver. In vitamin $\mathrm{K}$-deficient patients, a prolonged prothrombin time may be observed within a few days of starting antibiotics. This abnormality is, however, readily reversed by the parenteral administration of 5 to $10 \mathrm{mg}$ of vitamin $\mathrm{K}$, unless liver function is impaired. Thus, patients who are already deficient in vitamin $\mathrm{K}$ are at increased risk of bleeding, particularly if receiving MTT- or MTZcontaining antibiotics, which may further impair vitamin $\mathrm{K}$ metabolism.

Cefoperazone and ceftriaxone are excreted in large amounts via the biliary tract (in contrast to cefotaxime, ceftizoxime, and ceftazidime, which are eliminated primarily via the kidneys) and can produce both marked changes in the fecal flora and marked reductions in vitamin $\mathrm{K}_{2}$. The MTT side chain found on moxalactam and cefoperazone and the MTZ moiety found on ceftriaxone (Figure 1) can also inhibit the hepatic microsomal carboxylases that aid in the generation of catalytically active vitamin $K[7,14]$.

The MTT side chain of moxalactam is readily hydrolyzed, owing to the oxygen molecule in its secondary ring (Figure 1), which eventually results in the enterohepatic cycling of high concentrations of this entity.

Therefore, patients at risk of bleeding must be recognized, monitored, and given vitamin $\mathrm{K}$ either prophylactically or therapeutically (if bleeding has already occurred). Administration of fresh frozen plasma may also be used to restore clotting factors to normal, subsequent to the onset of bleeding.

\section{Platelet Dysfunction Induced by Antibiotics}

Some beta-lactam antibiotics irreversibly inhibit adenosine diphosphate-dependent platelet aggregation, a process that normally plays a key role in hemostasis [14]. This phenomenon is recognized by a prolongation of the bleeding time.

Both carbenicillin and moxalactam have been shown to markedly prolong the bleeding time of volunteers [15], a property that has been related to the alphacarboxyl side chain (Figure 1), which also enhances their anti-pseudomonal activity. There is a delay of two or three days after administration of moxalactam before the increased bleeding time becomes evident.

Prolonged bleeding may be detected or monitored in vitro by the technique known as platelet aggregometry, performed in the presence of adenosine diphosphate $[7,16]$, and has been found to depend on both the concentration of the antibiotic and the duration of treatment. Alpha-carboxylated beta-lactams, such as carbenicillin, ticarcillin, and moxalactam, have the greatest inhibitory effect on platelet aggregation. Other cephalosporins lack this side chain and have only slight effects on platelet function. Moxalactam (greater than $4 \mathrm{~g}$ per day for one week) consistently impairs platelet function and prolongs the bleeding time, whereas ceftriaxone and ceftizoxime $(6 \mathrm{~g}$ per day) have no demonstrable effect on platelet function [17].

In the presence of renal or hepatic dysfunction, many other antimicrobials may predispose to bleeding by this or related mechanisms. The "template bleeding time" technique appears to be the best way to monitor the bleeding tendency secondary to platelet dysfunction [18]. Treatment of the defect and of the bleeding it causes requires transfusion of fresh platelets and discontinuation of the causative agents; vitamin $\mathrm{K}$ administration does not appear to be helpful.

\section{HYPERSENSITIVITY REACTIONS}

The third-generation cephalosporins share major and minor antigenic determinants with penicillins. In vitro these compounds often cross-react with antibodies to various other penicillins or cephalosporins, but such antibody reactions do not appear to be useful as predictors of clinical allergic reactions [19].

Serious allergic reactions during treatment with cephalosporins are uncommon. Nonetheless, anyone with a history of penicillin allergy may develop a similar allergic reaction when given a cephalosporin, but a history of delayed allergic reactions to penicillins is only a relative contraindication to cautious administration of a third-generation cephalosporin. However, if immediate reactions (urticaria or anaphylaxis) to penicillins have occurred, it is prudent to avoid the use of any cephalosporin. Desensitization has been tried with success in a few such patients, but is not entirely reliable.

\section{IMMUNE MEDIATED HEMATOLOGIC REACTIONS}

All of the cephalosporins may cause immune hematologic reactions (Table III). Immune hemolytic anemia or thrombocytopenia may be caused when the cephalosporin acts as a hapten, which reacts with cellular proteins to form antigens that elicit antibody reactions. Such reactions may result from the participation of the antigen in formation of immune complexes that become attached in a nonspecific way to platelets, 
from the reaction of the antibody with cell-bound drug, from the induction of antibodies directed only against intrinsic cellular antigens, or from a combination of these mechanisms.

In the third-generation class, hemolytic anemias have been described only with ceftriaxone, although positive direct Coomb's antiglobulin tests can be seen in about 3 percent of patients receiving cephalosporins [20].

Leukopenia secondary to reversible suppression of the bone marrow has been reported after two to three weeks of high-dose therapy with cephalosporins. Characteristic features include depletion of bone marrow precursors, marrow cell proliferation, and immune-mediated destruction of granulocytes. If leukopenia becomes a problem, the blood count may be monitored, with discontinuation of the antibiotic therapy if the condition becomes severe; recovery is usually prompt.

Immune-mediated thrombocytopenia is rare with these antimicrobials, but has been reported with both ceftriaxone and cefotaxime $[20,21]$. In one study it was observed in 3.8 percent of recipients of cefotaxime, and a similar rate was observed in recipients of cefazolin [21]. Moxalactam, which has an oxygen atom rather than a sulfur in the secondary ring structure (Figure 1), is less likely to cross-react with the penicillin antibodies since it does not readily form sulfhydryl bonds. Thus, moxalactam is useful in cases in which a cephalosporin is required but in which there is a previous history of immune reactions to penicillin or other cephalosporins.

Some of the cephalosporins can adversely affect polymorphonuclear chemotaxis (cefoperazone), or suppress lymphocyte mitogenic responses (ceftriaxone), but the clinical significance of these observations is unknown [22].

\section{GASTROINTESTINAL SIDE EFFECTS}

Symptoms of the gastrointestinal side effects of third-generation cephalosporins are protean, and include nausea, vomiting, anorexia, abdominal cramps, and diarrhea. The characteristics of these side effects are shown in Table IV. They are usually benign or self-limited, and resolve rapidly after the drug treatments are discontinued. Many of these symptoms are often attributed, without much supporting evidence, to direct irritation of the intestinal mucosa by the antimicrobial. If so, they might be more frequent with moxalactam, cefoperazone, and ceftriaxone, the analogues of which are excreted to the greatest extent via the biliary tract, though no good clinical data exist to support this theory. It has been postulated that cefoperazone increases secretion of bile salts into the intestines, and that the resultant high concentration of bile salts causes diarrhea (Jacobson CE, personal communication). In addition, studies suggest that cefoperazone and ceftriaxone produce the most marked changes and cefotaxime the least, in the normal fecal flora, with selection of resistant pathogens. These changes are probably responsible for many of the adverse effects such as superinfection, diarrhea, and colitis [23-25].

In addition to impairing synthesis of vitamin $K_{2}$ in the colon, these changes in fecal flora favor intestinal superinfection or overgrowth with Enterococcus faecalis, Clostridium difficile (see the following section),

\section{TABLE IV}

\section{Characteristics of Gastrointestinal Side Effects of the Third} Generation Cephalosporins

- Nonspecific diarmea (dysbiosis), particularly with moxalactam, cefoperazone, and ceftriaxone

- Increased bile salt excretion with cefoperazone

- All third-generation cephalosporins can cause Clostridium difficile colitis (pseudomembranous colitis). Pseudo-epidemics may occur. Colitis sometimes begins after therapy is discontinued

- Overgrowth of resistant organisms

- Interference with vitamin K synthesis

and Candida. Resistant organisms in the intestines appear capable of transmitting genetic factors responsible for antimicrobial resistance via plasmids, so they are likely to be epidemiologically important in production of nosocomial infections caused by antibioticresistant organisms.

\section{Pseudomembranous Colitis Caused by $C$. difficile}

Toxigenic $C$. difficile can be found in the feces of more than 10 to 20 percent of hospitalized adults treated with antibiotics. Overgrowth of this organism may result in production of large amounts of its toxins, which have been implicated in the origin not only of diarrhea and pseudomembranous colitis, but also of toxic megacolon, severe diarrhea in Hirschsprung's disease, and worsening or relapse of diarrhea in patients with chronic inflammatory bowel diseases.

All the third-generation cephalosporins have been implicated in cases of $C$. difficile-induced pseudomembranous colitis, and the rates of this complication are not clearly higher for those antimicrobials excreted primarily via the biliary tract and found in high concentrations in feces. In premarketing trials, diarrhea of unspecified cause was arguably more common with cefoperazone (3.3 percent of treated patients) than with moxalactam ( 2.5 percent), ceftizoxime (1.8 percent), or cefotaxime (1.2 percent) [26]. This apparent paradox may be explained by the fact that even low fecal concentrations of antimicrobials (such as those reached when parenteral cephalosporins excreted primarily by the kidneys are also excreted to a lesser extent via the biliary route) are adequate to disturb the delicate balance in the fecal flora. In fact, antimicrobials like cefoperazone, which is found in high concentrations in feces, may inhibit growth of seemingly resistant $C$. difficile isolates in the intestinal tract until after the antibiotic therapy is discontinued, when the organism has an opportunity to multiply and cause disease. Such a delay in onset of the colitis makes it particularly difficult for clinicians to associate it with the inducing antibiotic.

Perhaps the most important point to remember about pseudomembranous colitis is that it may be seen, albeit only occasionally, with use of any of the third-generation cephalosporins.

\section{HEPATIC AND BILIARY SYSTEMS}

Mild, reversible abnormalities in liver function tests are not uncommon in patients receiving third-generation cephalosporins, but there have not been any reports of clinically evident toxicity or hypersensitivity.

Ceftriaxone has the unique property of causing sludge (pseudolithiasis) to form in the gallbladder, particularly in children $[27,28]$. None of the other 


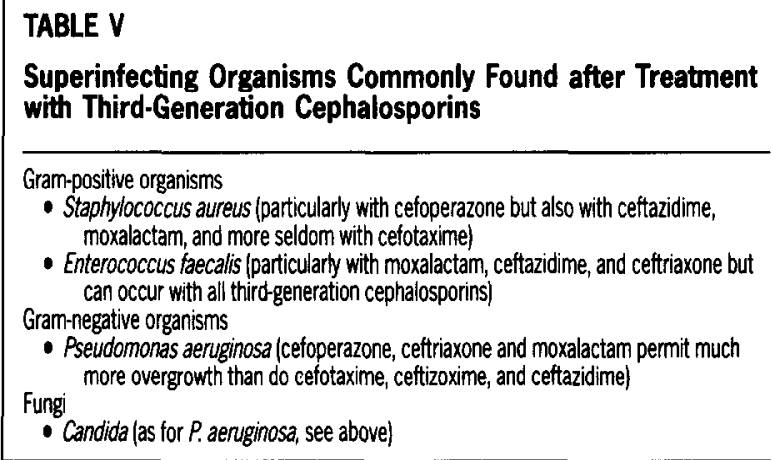

cephalosporins has been reported to cause this syndrome. Associated symptoms may include nausea, epigastric distress, colic, and tenderness to palpation or fist percussion in the right upper quadrant; the clinical picture resembles acute cholecystitis. The sludge is of uncertain composition, mobile, and best detected using ultrasonography. In a prospective ultrasonography study [27], 43 percent of 37 treated patients showed biliary "concrements" and one patient ( 3 percent) had urolithiasis. Three of 16 with gall bladder concrements also had biliary symptoms, which resolved within five days after discontinuation of ceftriaxone. The authors speculated that the abnormality was seen most commonly when ceftriaxone was given by intravenous bolus over three to five minutes. Cholecystectomy has been carried out on patients who have developed this syndrome, but there is increasing awareness of the syndrome and with it recognition that symptoms subside and the sludge gradually dissolves after the drug treatment is discontinued. Cholecystectomy is probably best avoided in these patients, but the drug treatment should be discontinued when these symptoms appear. Although this is a relatively benign side effect, it can be a disturbing one.

A high proportion (more than 90 percent) of circulating ceftriaxone is bound to serum proteins and consequently may displace bilirubin from protein binding sites, thus causing a slight hyperbilirubinemia $[29,30]$. As a result, there may be an increased frequency of the complications of hyperbilirubinemia in neonates (particularly those already hyperbilirubinemic) treated with ceftriaxone. Ceftriaxone should be avoided in all such cases.

At present, there is insufficient knowledge of the problems of biliary sludge and hyperbilirubinemia. It is not known whether the hyperbilirubinemia is related in any way to the mechanism of formation of gallbladder sludge, but it is apparent that ceftriaxone has some unique side effects relative to the biliary tract that are worthy of further investigation.

\section{SUPERINFECTIONS}

The broad spectrum of antibacterial activity of the third-generation cephalosporins can result in profound changes in the normal flora at various sites in the body, particularly the gastrointestinal tract, vagina, skin, and upper respiratory tract. If resistant organisms increase greatly in numbers at those sites, an increase in both mucocutaneous or systemic infections by opportunistic organisms may be the result. The acquisition of antibiotic-resistant pathogens from exogenous nosocomial sources may also be facilitated by use of these antibiotics. Although colonization is more frequent than infection with such organisms, it is often difficult to distinguish between colonization and superinfection, and unnecessary and expensive changes in antibiotic therapy may then result.

The third-generation cephalosporins were designed primarily to have good activity against gram-negative bacilli, but this was accompanied by a lesser degree of activity against staphylococci and enterococci. This differential antibacterial activity is particularly evident with ceftazidime, moxalactam, and cefoperazone, which are much less active than cephalothin or cefazolin against these staphylococci and enterococci. In vitro, cefotaxime has the best antistaphylococcal activity of all third-generation cephalosporins (Fekety and Cimino, unpublished observations) [31]. Ceftriaxone has almost equivalent activity, except in the presence of serum (as a result of the high affinity of ceftriaxone for serum proteins). These findings may be of importance for treatment of neutropenic patients with staphylococcal infections, in whom good antibacterial activity may play a decisive role. None of the cephalosporins is active against methicillin-resistant staphylococci, and if used in treatment of these infections, there is a high clinical failure rate.

The reported incidence of superinfections during therapy with parenteral cephalosporins ranges from 1 to 15 percent [32]. These are caused most often by enterococci, very resistant gram-negative bacilli such as Pseudomonas, and fungi such as Candida (Table V). Those antibiotics with the least biliary excretion (cefotaxime, ceftizoxime, and ceftazidime) appear to result in less overgrowth by resistant organisms in stools than do those agents, such as cefoperazone, ceftriaxone, and moxalactam, that are excreted in bile in large amounts.

Resistance of gram-negative bacilli to the thirdgeneration cephalosporins may be mediated by inducible (chromosomal) beta-lactamases. These can become stably depressed, and resistance may continue after the inducing drug treatment has been discontinued. Pathogens most likely to have this ability include Pseudomonas aeruginosa, Enterobacter cloacae, Citrobacter, Serratia, Proteus spp., Providencia, and Acinetobacter.

Enterococcal superinfections have been reported most often in patients receiving moxalactam ( 2 to 8 percent of cases), but they have also been reported less commonly in patients treated with ceftizoxime, ceftazidime, and cefotaxime [32,33], with the lowest incidence reported for cefotaxime [34].

\section{NEPHROTOXICITY}

Cephalosporins have the capacity for causing renal injury as a result of hypersensitivity-induced interstitial nephritis, by direct toxicity to the renal tubules, or by synergistic action with other nephrotoxic compounds, such as aminoglycoside antibiotics or loop diuretics. In theory, any cephalosporin can induce interstitial nephritis, but significant nephrotoxicity has not yet been reported with any of the third-generation cephalosporins. In addition, there is no convincing evidence that any third-generation cephalosporins potentiate the nephrotoxicity of aminoglycosides or loop diuretics. There are a few reported cases of mild renal impairment occurring following use of ceftriaxone and ceftazidime, and one of the reports urged caution in the use of ceftazidime in patients with impaired renal function or in combination with aminoglycosides $[33,35]$. However, there is no good evidence that any 
cephalosporin other than cephaloridine (which is no longer available in the United States) is nephrotoxic. In rabbits, extremely large doses of either moxalactam or cefotaxime caused no detectable renal abnormalities, and in rats, ceftazidime actually reduced the nephrotoxicity of gentamicin [36].

There is no good clinical evidence for or against a synergistic toxicity of the third-generation cephalosporins with other nephrotoxins, such as aminoglycosides. Overall, it appears that these cephalosporins have a low nephrotoxic potential, in marked contrast to the aminoglycosides [37]. This is a major argument for the use of third-generation cephalosporins rather than aminoglycosides in many patients with serious infections.

\section{NEUROTOXICITY}

Headaches, paresthesias, and other mild central nervous system side effects have been reported with all third-generation cephalosporins. Seizures have been reported in patients treated with ceftazidime, but not with cefotaxime, ceftizoxime, or ceftriaxone [38]. All the third-generation cephalosporins other than cefoperazone show marked penetration across the blood brain barrier, particularly in cases of meningeal inflammation. Thus it is possible that most cephalosporins have the capacity to occasionally cause seizures, if given in doses sufficient to produce high concentrations in serum and brain.

\section{DISULFIRAM-LIKE REACTIONS}

Disulfiram-like (Antabuse) reactions consist of a variety of symptoms, as shown in Table VI. They occur in patients taking disulfiram who ingest alcoholic beverages. These reactions can also be produced by consuming ethanol while receiving certain cephalosporins, notably those containing the MTT side chain at the 3-position of the nucleus (Figure 1). The MTT side chain has structural similarity to disulfiram and it is thought to cause these reactions by a similar mechanism, namely by binding to and interfering with the action of the liver enzyme aldehyde dehydrogenase, which metabolizes the acetaldehyde breakdown product of ethanol to water and carbon dioxide. Accumulation of acetaldehyde is thought to cause the symptoms. There is a notable time lag between administration of the cephalosporin and susceptibility to the reaction after subsequent ingestion of ethanol. This is consistent with the notion that a metabolite of the antibiotic is important in causing the reaction. It has also been suggested that the time required for release of the free MTT side chain or its attachment to the enzyme may account for the lag period [39]. Medications, such as elixirs and cough syrups containing alcohol, have been implicated in some cases of disulfiram-like reactions.

Moxalactam and cefoperazone are the analogues most likely to cause these reactions. There is one report of a disulfiram-like reaction in a patient given ceftriaxone. In this case, it is possible that the MTZ group of the ceftriaxone molecule was metabolized to a compound resembling the moxalactam or cefoperazone MTT group metabolite [40].

\section{COMMENTS}

Third-generation cephalosporins are remarkably safe antimicrobials, particularly when compared with the aminoglycosides. However, they can occasionally

\section{TABLE VI}

Characteristics of Third-Generation Cephalosporin-Induced Disulfiram-Like (Antabuse) Reactions with Ethanol

\author{
- Related to the MTT side chain of moxalactam and cefoperazone \\ - One case has been demonstrated with ceftriaxone (MTZ side-chain) \\ - Due to blockade of alcohol dehydrogenase \\ - Symptoms \\ Weakness \\ Headache \\ Blurred vision \\ Vertigo \\ Nausea and vomiting \\ Confusion \\ Warmth, sweating, and flushing \\ Tachycardia \\ Hypotension \\ - Delayed onset of symptoms after ingestion of antibiotic
}

produce serious reactions. Bleeding, superinfections, disulfiram-like reactions, and pseudomembranous colitis are the most important complications. Although the rate of adverse reactions does not vary greatly among the third-generation agents, cefotaxime and ceftizoxime appear to be associated with the lowest frequency of serious side effects, and moxalactam is associated with the highest frequency, primarily as a result of a unique propensity to cause coagulopathies and bleeding. Considering their broad antibacterial spectrum and low frequency of adverse reactions, the third-generation cephalosporins are useful alone or in combination with other antimicrobials for treatment of serious infections in hospitalized patients.

\section{REFERENCES}

1. Platt R: Adverse effects of third-generation cephalosporins. J Antimicrob Chemothe 1982; 10 (suppl C): 135-140.

2. Meyers BR: Comparative toxicities of third-generation cephalosporins. Am J Med 1985; 79: $96-103$.

3. Andrassy K, Bechtold H, Ritz. E: Hypoprothrombinemia caused by cephalosporins. J Antimicrob Chemother 1985; 15: 133-135.

4. Jones RN: Hemorrhagic complications of cephalosporin therapy. Antimicrobic Newsletter 1988; 12: 85-89.

5. Sattler FR, Weitekamp MR, Sayegh A, Ballard J0: Impaired hemostasis caused by betalactam antibiotics. Am J Surg 1988; 155: 30-39.

6. Kerremans AL, Lipsky JJ, Van Loon J, Gallego MO, Weinshilboum RM: Cephalosporin induced hypoprothrombinemia: possible role for thiol-methylation of 1-methyltetrazole-5. thiol and 2-methyl-1,3,4-thiadiazole-5-thiol. J Pharm Exp Ther 1985; 235: 382-388. 7. Bang NU, Tessler SS, Heidenrich RO, Marks CA, Mattler LE: Effects of moxalactam on blood coagulation and platelet function. Rev Infect Dis 1982; 4: S546-S555.

8. Andrassy K, Koderisch J, Fritz S, Ritz E: New beta-lactam antibiotics and hemorrhagic diathesis-comparison of moxalactam and cefotaxime. Clin Ther 1983; 6: 34-42.

9. Brown RB, Klar J, Lemeshow S, Teres D, Pastides H, Sands M: Enhanced bleeding with cefoxitin or moxalactam: statistical analysis within a defined population of 1493 patients. Arch Intern Med 1986; 146: 2159-2164.

10. Andrassy $K$, Koderisch J, Fritz S, Bechtold $H$, Sonntag $H$ : Alteration of hemostasis associated with cefoperazone treatment. Infection 1986; 14: 33-37.

11. Haubenstock A Schmidt P, Zazgornik J, Baicke $P$, Kopsa H: Hypoprothrobinaemic bleeding associated with ceftriaxone. Lancet 1983; : 1215-1216.

12. Friedman PA: Vitamin K-dependent proteins (editorial). N Engl J Med 1984; 310: 14581460.

13. ConRamotar K, Conly JM, Chubb H, Louie T: Production of menaquinones by intestinal anaerobes. J Infect Dis 1984; 150: 213-218.

14. Aber RC, Weitekamp MR: Coagulation defects. In: Karchmer AW, ed. Safety of parenteral cephalosporins-a clinical review. Little Falls, New Jersey: National Infectious Diseases Information Network, 1986; 9-12.

15. Weitekamp MR, Caputo GM, AI-Mondhiry HR, Aber RC: The effects of latamoxef, cefotaxime, and cefoperazone on platelet function and coagulation in normal volunteers. Antimicrob Chemother 1985; 16: 95-101.

16. Shattil SJ, Bennett JS, McDonough M, Turnbull J: Carbenicillin and penicillin $G$ inhibit platelet function in vitro by impairing the interaction of agonists with the platelet surface. Clin invest 1980; 65; 329-337.

17. Weitekamp MR, Aber RC: Prolonged bleeding times and bleeding diathesis associated with moxalactam administration. JAMA 1983; 249: 69-71.

18. Joehl RJ, Rasbach DA, Ballard JO, Weltekamp MR, Sattler FR: Moxalactam. Evaluation of clinical bleeding in patients with abdominal infections. Arch Surg 1983; 118: 12591261 . 
19. Blanca $M$, Fernandez J, Miranda $A$, et at: Cross-reactivity between penicillins and cephalosporins: clinical and immunologic studies. J Allergy Clin Immunol 1989; 83: 381 385

20. Petz LD: Immunologic cross-reactivity between penicillins and cephalosporins: a review. J Infect Dis 1978; 137: S74-S79.

21. Smith CR: Cefotaxime and cephalosporins: adverse reactions in perspective. Rev Infect Dis 1982: 4 (suppl): S481-\$488.

22. Fietta A, Mangiarotti P, Gialdroni Grassi G: Chemotherapeutic agents: aspects of their activity on natural mechanisms of defense against infections. Int J Clin Pharm Ther Toxicol 1983; 21: 325-338

23. Arvidsson A, Alván G, Angelin B, Borgå O, Nord CE: Ceftriaxone: renal and biliary excretion and effect on the colonic microflora. J Antimicrob Chemother 1982; 10: 207215.

24. Guggenbichler JP, Kofler J: Influence of third-generation cephalosporins on aerobic intestinal flora. J Antimicrob Chemother 1984; 14 (suppl B): 67-70.

25. Guggenbichler JP, Kofler J, Allerberger $F$ : The influence of third-generation cephalosporins on the aerobic intestinal flora. Infection 1985; 13 (suppl 1): S137-S139.

26. Fekety R: Gastrointestinal side effects. In: Safety of parenteral cephalosporins-a comparative review. Karchmer AW, ed. Little Falls, New Jersey: National Infectious Diseases Information Network, 1986; 17-22.

27. Schaad VB, Wedgwood-Krucko J, Ischaeppeler H: Reversible cetriaxone-associated biliary pseudolithiasis in children. Lancet 1988; Il: 1411-1413.

28. Jacobs RF, Kearns GL: Ceftriaxone-associated cholelithiasis. Pediatr Infect Dis J 1988 7: $434-436$.

29. Gulian JM, Dalmasso C, Pontier F, Gonard V: Displacement effect of ceftriaxone and bilirubin bound to human serum albumin. Chemotherapy 1986; 32: 399-403.

30. Wadsworth SJ, Suh B: in vitro displacement of bilirubin by antibiotics and 2 . hydroxybenzoylgiycine in newborns. Antimicrob Agents Chemother 1988; 32: 1571-1575. 31. Jones RN, Barry AL, Packer RR: The activity of cefotaxime and desacetyl cefotaxime alone and in combination against anaerobes and staphylococci. Diagn Microbiol Infect Dis 1984: 2 : 375-465.

32. Glew RH: Superinfections. In: Karchmer AW, ed. Safety of parenteral cephalosporinsa clinical review. Little Falls, New Jersey: National Infectious Diseases Information Network, 1986; 27-30.

33. Eron Lل, Goidenterg RI, Parke CH, Poretz DM: Ceftazidime therapy of serious bacterial infections. Antimicrob Agents Chemother 1983; 23: 236-241.

34. Jones RN: Gram-positive superinfections following beta-lactam chemotherapy: the significance of the Enterococcus. Infection 1985; 13 (supp| 1): S81-S88.

35. Alestig K. Trollfors B, Andersson R, Olaison L, Suurkula M, Norrby SR: Ceftazidime and renal function. J Antimicrob Chemother 1984; 13: 177-181.

36. Capel-Edwards $K$, Pratt DAH: Renal tolerance of ceftazidime in animals. J Antimicrob Chemother 1981; 8 (suppl B): 241-245.

37. Kahlmeter G, Dahlager J: Aminoglycoside toxicity - a review of clinical studies published between 1975 and 1982. J Antimicrob Chemother 1984; 13 (suppl A): 9-22.

38. Physicians Desk Reference, 43rd ed. Little Falls, New Jersey: Medical Economics Company, 1989; 994-997.

39. Buening MK, Wold JS: Ethanol moxalactam interactions in vivo. Rev Infect Dis 1982; 4 (suppl): 555-563.

40. Moskovitz BL: Clinical adverse effects during ceftriaxone therapy. Am J Med 1984; 77 84-88. 